\title{
Correction: Brolucizumab—early real-world experience: BREW study
}

\author{
Ashish Sharma (1) - Nilesh Kumar (1) - Nikulaa Parachuri - Srinivas R. Sadda - Giulia Corradetti (1) - Jeffrey Heier • \\ Adam T. Chin (1) - David Boyer - Pouya Dayani - Sruthi Arepalli - Peter Kaiser (1)
}

Published online: 15 December 2020

(c) The Royal College of Ophthalmologists 2020

Correction to: Eye

https://doi.org/10.1038/s41433-020-1111-x

Since online publication of this article, the authors became aware of two errors in the text of the manuscript:

1. Page 1 Paragraph 1, an incorrect approval date was inserted. The correct sentence is provided below:

Brolucizumab (Beovu, Novartis, Basel, Switzerland) is the newest anti-vascular endothelial growth factor (anti-VEGF) drug. It was approved for the treatment of neovascular age-related macular degeneration (nAMD) by the US-FDA on October 7, 2019, followed by the European Commission's approval for use in the European Union on February 13, 2020.

2. Page 2 Paragraph 1, an incorrect percentage was provided. The correct sentence is provided below:

Pigment epithelial detachment (PED) was present in 31 eyes (73.8\%) at baseline and resolved in 2 eyes (6.4\%), was reduced in 13 eyes $(41.9 \%$, and did not show any change in 16 eyes (51.6\%) (Fig. 1).

The authors apologise for any inconvenience caused. 